\title{
Theoretical aspects of optimization synchronous machine rotors
}

\author{
Timur Petrov ${ }^{1, *}$, and Alfred Safin ${ }^{1}$ \\ ${ }^{1}$ Kazan State Power Engineering University, Kazan, Russia
}

\begin{abstract}
Permanent magnet synchronous electric machines are increasingly used in various drive technical complexes (oil industry, small generation, aviation industry, etc.). The presented optimization allows you to increase the energy performance of a synchronous machine without increasing the overall dimensions. Permanent magnet synchronous motors have become much more commonly operated in various drive technical complexes. For each drive complex, it is necessary to fulfil the requirements for the developed torque, cooling conditions and strength characteristics. The distribution of materials (topology) in the rotor casing of a synchronous machine (permanent magnets, iron, air, etc) determines the characteristics of permanent magnet synchronous electric machine.
\end{abstract}

\section{Introduction}

Topological optimization was initially carried out on the basis of a numerical method for solving partial differential equations, which was necessary to reduce mass and increase the strength characteristics of machine structures.

The proposed method allows you to transfer topological optimization to electromagnetic and thermal processes in a synchronous machine in order to increase the energy characteristics and reliability of the electric machine for a given mass and dimension indicators.

At the moment, there are no works on integrated topological optimization of rotors of synchronous electric machines with permanent magnets.

The algorithm of step-by-step topological optimization of rotors taking into account electromagnetic, thermal processes and strength calculation will allow designing prototypes of rotors with high energy characteristics for the preparation of conceptual and working design documentation.

The project aims to create a software package that will be used at electrical enterprises producing drive systems based on synchronous electric machines with permanent magnets.

The paper proposes a modern method of designing and optimizing synchronous motors with permanent magnets through the use of genetic algorithms. Operation of synchronous machines instead of asynchronous for electric drive sucker-rod pumping units will make it possible to obtain higher parameters of energy efficiency, and also synchronous motors have an extremely compact design. The structure of synchronous motors makes them much smaller and lighter than similar induction motors. A synchronous machine is up to 40 percent more compact than a similar induction motor. They correspond to a size that is two standard sizes smaller, which makes it possible for a more compact machine design. In addition, synchronous motors weigh 50 percent less and its inertia is 60 percent lower than that of a similar induction motor.

Designing an electric machine as a heuristic process does not guarantee a better solution. Methods are needed to complement the designer's experience and intuition in order to find the optimal (rational) solution. Design optimization is currently achieved by calculating the minimization of the functional of the variational problem. The proposed method allows you to transfer topological optimization to electromagnetic processes in synchronous motors to determine the directivity angle of the coercive force of the magnets used in the machine, the number of phases and turns of the windings, the size of the stator grooves and permanent magnets in order to increase the characteristics (power factor, reliability) of the electric machine at specified mass and size parameters.

\section{Literature review}

In Russia, the active use of topological optimization methods has been introduced relatively recently, and is mainly used to increase the specific strength of structures in the aerospace industry [1], optimization of turbine blades [2] or pistons of internal combustion engines [3]. The features of all these works are the topological optimization of only one material and the maximization of only one objective function (in most works of the Russian-speaking segment this is an increase in strength with a decrease in weight).

In foreign sources, work on topological optimization has been ongoing for a long time, and both strength parameters and the values of the electromagnetic and thermal fields are increasing.

The article [4] demonstrated the advantages of topological optimization of several materials compared

* Corresponding author: tobac15@ mail.ru 
to standard optimization (one material). The proof was the optimized design of the automobile engine for both optimization cases, and in the first case, a more rigid design with the same weight is obtained. The difference from our topic is that we will optimize synchronous motors with permanent magnets, and taking into account not one controlled parameter (weight in the article), but the electromagnetic, thermal and strength component, and this will allow us to obtain the most efficient motor design.

For example, an article is presented [5], in which the design of an electric motor without DC brushes is optimized (namely, the rotor configuration) based on the finite element method to increase specific power by increasing the electromagnetic field.

In [6], a two-dimensional model was considered for determining the electromagnetic field in the air gaps of synchronous motors with permanent magnets, and in scientific work [7] a method for designing permanent magnets that can already be used to obtain optimal designs is shown. In the article [8] a variant of topological optimization was presented through the use of a genetic algorithm, and in [9] optimization was achieved as a result of using the finite element method. In these works, optimization was necessary to increase the values of the electromagnetic field; thermal calculation was presented in [10], where an engine with the highest possible heat flux was designed. The design of engines can be made more efficient by using topological optimization based on sensitivity [11]. To increase the productivity of the genetic algorithm, optimal initial populations are necessary; work [12], which shows an algorithm for generating a population with high values of characteristics, can cope with this. The development of the genetic algorithm can also lie in the direction of adaptive grid changes right during optimization [13]; the use of the sequential linear programming method [14] is also an interesting solution.

In [15], an option was considered using the ON / OFF sensitivity method to optimize the design of the rotor. The following document [16] shows an interesting joint method for optimizing the design of the rotor, combining topological optimization and the limitations associated with the shape of the magnets.

It is worth noting that at the moment there are no works on complex topological optimization of permanent magnet synchronous motors, which allows taking into account the influence of the distribution of materials on the electromagnetic, thermal and strength characteristics of machines, in order to choose a rational design option.

\section{Theoretical aspects of topological optimization}

Topological optimization is one of the ways to find optimal solutions to structural problems for a particular structural model, including for electrical equipment designs.

This type of optimization is mainly used at the conceptual stage of design. The optimized function is the value of the potential energy of the system, as well as the movement of nodes. To solve the problems posed by this type of optimization, different methods of mathematical modeling are used. It is important to note that finding the exact solutions to topological optimization problems is most often difficult. And, therefore, in practice, algorithms for approximate numerical solutions are developed. In a broad sense, topological optimization can be classified as follows:

1) discrete element;

2) continuous.

The discrete-element type of optimization implies such an approach to design, in which there will be a ready-made set of possible discrete elements for the considered structural model. By changing the geometric parameters of any element from zero (the element disappears) to the maximum possible, the final structure will have different sizes and topologies. Continuous topological optimization is designing as a kind of continuous "void" or material with extremely low density values. By changing the distribution ratio, the "void" / material or density values within the maximum possible small values, the size value and the topology of the structural model of the object under consideration.

The distribution of materials in the rotor is one of the most important factors determining the magnetostatic field of the machine, thermal characteristics and determining the strength characteristics in electric machines.

In addition to commonly used materials such as steel sheet, permanent magnets (neodymium-ferrum-boron, samarium-cobalt), some recently developed magnetic materials, such as soft magnetic composites, amorphous and grain-oriented silicon steel can exhibit more high magnetic field parameters, for example, low losses and high values of saturation density, which leads to lower operating costs. Thus, we can say that the use of these types of magnets will allow the development of synchronous motors with the most optimal characteristics, both from a technical and economic point of view.

The production methods used, obviously, also have a fundamental role in the life cycle of developing synchronous motors, since they directly affect the quality of workmanship and actual performance.

It is important to note that in order to obtain the optimal characteristics of synchronous motors, the rotor can have such a complex configuration that it is difficult to manufacture, and this is a serious drawback.

Another disadvantage is that the characteristics of the materials in question will depend on the production methods. Optimum parameters can only be obtained with a full understanding of the theoretical aspects of magnets used for the design of synchronous machines, and accordingly this knowledge should affect production.

This must be taken into account when configuring the parameters of topological optimization.

Currently, the industrial production of permanent magnets with high energy performance is developing. Such magnets make it possible to create a large magnetic flux in small volumes, allowing one to significantly increase the ultimate power. 
The most important requirements for engines in the drive mechanisms of rocking machines are:

- high energy-dynamic parameters;

- small dimensions and weight.

To achieve maximum values of the energy and dynamic parameters of synchronous motors with small dimensions, high-energy permanent magnets are used.

This allows:

- firstly, reduce the volume of permanent magnets;

- get the smallest moment of inertia of the rotating part of the motor rotor;

- thirdly, to obtain a greater magnetic flux, that is, large values of the starting, electromagnetic moment, specific power and efficiency.

Currently, permanent magnet synchronous motors use high-energy magnets based on samarium and cobalt and hard magnetic materials based on a neodymiumiron-boron alloy.

To use promising types of materials in the construction of an electric motor, it is important to emphasize that it is necessary to focus on the development of promising engine topologies based on new algorithms and topological optimization methods.

Thus, the rational design of the engine being designed should be acceptable in terms of manufacturability and energy performance.

The following algorithm of complex topological optimization is proposed (for example, a synchronous motor with permanent magnets and an internal rotor, the stator design and stator current are assumed to be given).

\section{Calculation algorithm}

Stage 1.

At this stage of topological optimization, the maximum torque on the shaft is determined.

The rotor design area, which is a cross section (axisymmetric task), is divided into a network of cells finite elements with the material inherent in each cell (for example, air, steel, types of magnets with different directions of magnetization, which are designated $0,1,2$, $3,4,5$, etc.).

Each topology is encoded with a unique code. Based on the genetic algorithm, the best solution is sought. Two initial samples are randomly selected. Next, several cells are selected for exchange by homogeneous crossing and, as a result, two new samples are created.

New samples inherit the best characteristics of previous samples, then the process repeats. A procedure has been proposed for cleaning materials to obtain homogeneous structures in the design area (for example, without air cavities in magnets), which makes it possible to simplify the manufacture of the prototype.

2 stage.

The level of thermal loads determines the ultimate impact on the material of permanent magnets, the insulation of the wires of the windings, bearing units, as well as other areas, limiting the life of the engine.

To reduce the heat load in the cross section of the prototype of the rotor obtained in stage 1, areas of ventilation ducts with dimensions are introduced to ensure the manufacturability of the manufacture of sheets of the rotor or its casting. For the same reason, the areas of the possible distribution of ventilation ducts must be symmetrical with respect to the radial guides and the minimum distance between the ventilation ducts and other materials is prescribed in the algorithm.

The first iteration sets the minimum possible size of ventilation ducts. The search for the best solution is based on a genetic algorithm, similar to stage 1 . The thermal field of the 3D model is calculated (due to the uneven distribution of temperatures) of the electric machine based on the obtained topology, to determine the maximum temperatures in the rotor housing.

3 stage.

The obtained optimal solution of the thermal regime, taking into account the indicated limitations, is directed to the strength calculation, taking into account the maximum rotor speed and mechanical properties of the materials of the designed rotor. If the results are unsatisfactory, the prototype returns to stages 1 and 2 .

Stage 4

The prototype of the first three stages is calculated torque on the shaft. If the received torque is not lower than $5 \%$ of the maximum torque obtained in stage 1 , the optimization result is considered achieved. An unsatisfactory result is excluded from the list of possible solutions, and the calculation is repeated from stage 1 .

A review of the scientific and technical literature, Internet resources, and data from manufacturers of electric machines shows that today there is no software package that implements complex topological optimization of the rotor of electric machines.

Due to the growing computational capabilities, direct numerical simulation by the finite element method today allows us to study in detail the various topologies of rotors with various types of materials (permanent magnets, steel, etc.), in order to determine the structure of the rotor that provides maximum torque, permissible thermal conditions and strength characteristics.

The creation of $3 \mathrm{D}$ prototypes of synchronous motors with specified parameters based on the results of integrated topological optimization, as well as full-scale models of electric motors and subsequent experimental studies allow us to create new engineering solutions with improved performance for various operating conditions.

The new ones are identification with a unique binary code of each element of the rotor of an electric motor, including various versions of the material used (materials of permanent magnets, steel grades, etc.) with specified properties, which allows you to calculate the necessary topology of an electric machine through an automated process, a procedure has been developed purification of materials to obtain homogeneous structures in the designed area (for example, without air cavities in magnets and steel), which allows to improve manufacturability prototype manufacturing.

The introduction into the topology of the areas of ventilation ducts and their calculation allows us to optimize the thermal conditions of the electric motor. 


\section{Modeling synchronous machines at the system level}

Currently, developers of electric machines use finite element methods to optimize parameters such as torque, efficiency, and induced voltage. Using FEM tools, for example, ANSYS Maxwell, allow developers to make design changes to the geometry, properties of materials, electrical characteristics of power supplies, use mechanical load to optimize the magnetic system of an electric machine. Nevertheless, it is necessary to consider the interaction of an electric machine with the electrical and mechanical components of the entire system, which affects the behavior of the controller. To this end, the magnetic system of the machine design must be tested and optimized throughout the entire drive system, including: - Batteries and DC link capacitors. · Power electronics. - Mechanical components of the drive. - Controllers and software. ANSYS Simplorer provides a state-of-the-art platform for comprehensive drive research. To model the unsteady, dynamic behavior of a machine in a system model, it is necessary to represent it at the level of an equivalent circuit. It should be noted that direct non-stationary magnetic calculation of the finite element model under the control of the simulator also takes place and the solution provides high-precision results, including all the effects of a dynamically changing magnetic field. However, the cost of simulation time when calculating the dynamics of a control system with mechanical loads and a finite element engine model together can be prohibitive. On modern computing equipment, it may take several days to solve such a problem. The method of extracting equivalent circuits generates a descriptive model of a three-phase synchronous machine of the system level based on the results of magnetic finite element analysis, which allows to reduce the simulation time by the above method several times. ECE models cover basic nonlinear magnetic saturation effects and groove effects. The resulting model is determined by a set of nonlinear differential equations describing the relationship between the phase currents of the machine and the magnetic flux.

Using the ECE model, a number of typical tasks can be solved:

- Harmonic analysis of voltage / current, moment;

- Dynamics of the control system;

- Short circuit protection.

The ECE technique was implemented for a threephase electric machine, presented in the non-stationary model ANSYS Maxwell 2D. The method can work with six-phase machines and / or 2D / 3D models.

To extract the parameters of the ECE model, the procedure requires a non-stationary three-phase model of the ANSYS Maxwell 2D engine, as source data. The ANSYS Maxwell model must contain at least three windings and at least four windings for machines with a phase rotor (including an excitation winding). The existing axial symmetry is taken into account. A custom user interface tool is used, where the user enters data on the basic topology of the machine (number of poles, number of slots, maximum current, etc.) The ANSYS Distributed solve (DSO) feature for distributed solution of a parametric task within the local network will help to speed up the process significantly. computers or highperformance cluster. As a result of a series of calculations, a summary table is created of the dependence of the magnetic flux on the stator current, excitation current (for the phase rotor) and the position of the rotor.

\section{Designed stand}

The principle of operation of the developed genetic algorithm is as follows.

An example of calculating the electromagnetic moment in the Elcut program is presented for an engine with axial magnets.

In accordance with the current documentation for a permanent magnet synchronous motor, the motors are tested according to the following program:

1. Measurement of the resistance of the windings at constant current in a practically cold or heated state;

2. Test at reduced speeds under load;

3. Determination of idle speed characteristics;

4. Determination of losses and efficiency

The equipment that was used to create the test bench is presented:

1. The control cabinet (the element is composite, therefore it will be considered in more detail).

2. The load engine AIR90L2 $2.2 \mathrm{~kW}$.

3 . The torque sensor M40-150.

M40 digital type torque sensors are designed to measure torque in a wide range of nominal values from $0.1 \mathrm{Nm}$ to $300 \mathrm{kNm}$ and can be used at rotational speeds up to $20,000 \mathrm{rpm}$.

Structurally, the sensors are made in the form of two separate components: a rotor and a stator, between which there are no brush contacts and bearings. The rotor incorporates an elastic strain element, slightly sensitive to the effect of bending moment, transverse and axial forces.

A microprocessor electronic module located on the rotor converts the strain gauge signals into a digital code, which is transmitted from the rotor to the stator via a non-contact telemetric communication channel. The digital encoded signal has a high noise immunity, provides high measurement accuracy, can be transmitted over considerable distances without distortion and loss of information.

The stator has a composite structure, which provides ease and simplicity of mounting the sensor on the test object.

M40 sensors are equipped with an optoelectronic speed sensor.

4. Compensation coupling MK-0.1.

MK disk couplings are designed to compensate for axial, radial, angular displacements, temperature deformations that occur during installation and during operation of torque sensors. MK couplings have significant axial and angular compliance, with high torsional stiffness. MK couplings are universal and can 
be used in various fields of mechanical engineering for transmitting torque between rotating shafts having misalignments and distortions of the axes.

5. Test engine valve.

In our case, we will use a synchronous permanent magnet motor with a power of $350 \mathrm{~W}$ BM1418 ZXF 350 W $48 \mathrm{~V}$, efficiency> 75\%, 40X40X30 cm, $12.0 \mathrm{~kg}$., Rated speed: $2800 \mathrm{rpm}$.

6. Speed sensor LA8.

A suitable sensor is a prerequisite for a successful solution to the problem. The A5S Series speed sensors are based on the robust differential Hall effect. Their lower limit of $0 \mathrm{~Hz}$ allows you to control the machine up to a complete stop. Non-contact, wear-free, maintenance-free and insensitive to scattered magnetic fields and machine vibrations. Other sensors, such as MPU (magnetic induction) or static Hall sensors, unlike the sensors used on the differential Hall effect, are sensitive to interference and therefore not suitable for applications with strict requirements.

Each series of sizes is offered with different lengths and diameters, as well as with different types of connection, but all these sensors have the same functional characteristics.

7. DKC control cabinet rack.

DKC two-frame mounting racks are designed for installing network and telecommunication 19 "equipment inside office and industrial premises. It is most advisable to install racks in special rooms or halls where there are common ventilation and air filtration systems.

\section{Optimization Research Results}

As a result of optimization, the rotor sector (45 degrees) will look like this (Figure 1), where the filled squares are magnetic material, which will increase the engine torque (for each engine this value is different, in our case it was about $7 \%$ ), with the same volume of magnets. But there are a few comments that still do not allow us to talk about the final optimization of the rotor:

1. It is necessary to optimize the thermal characteristics of the rotor (at least add an element such as air).

2. It is necessary to conduct a strength calculation of the resulting design of the rotor. These calculations will be carried out in the following articles.

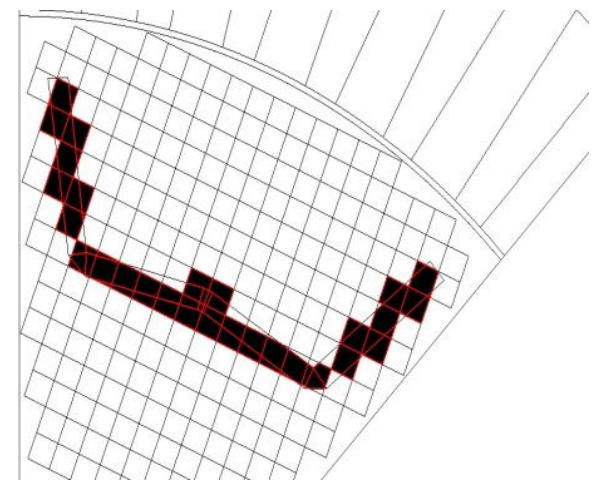

Fig. 1. Sector of the rotor after the optimization.

\section{Conclusion}

In this paper, the following results were obtained:

1. The analysis of modern scientific, technical, regulatory, methodological literature on the development and creation of synchronous motors with permanent magnets showed that they are currently being used, but there are still opportunities for the development of these machines, from the point of view of improving energy efficiency and optimization.

2. Currently, the industrial production of permanent magnets with high energy performance is developing. Such magnets make it possible to create a large magnetic flux in small volumes, allowing one to significantly increase the ultimate power of electric machines.

An analytical information search indicates that one of the most promising areas is the use of topological optimization for synchronous electric machines, as it is more conceptual and applied. The optimization of the topology of synchronous electric machines allows you to convert its geometric and shaped parameters, for example, the dimensions of the rotor grooves, which will lead to a harmonious distribution of the magnetic flux, which means less heating of the insulation and rotor windings.

\section{Acknowledgements}

The reported study was funded by RFBR, project number 1937-90134.

\section{References}

[1] K.A. Bashin, R.A. Torsunov, S.V. Semenov, Methods of topological optimization of structures used in the aerospace industry, Bulletin of the PNRPU. Aerospace engineering 4, 51 (2017)

[2] B.E. Vasiliev, L.A. Magerramova, Analysis of the possibility of using topological optimization in the design of uncooled turbine blades, Vestnik SGAU, 3-1 (2015)

[3] L.L. Myagkov, S.M. Sivachev, E.E. Strizhov, S.P. Chirsky, Topological optimization of a highperformance diesel piston, Dvigatelestroyeniye, 2, 3-10 (2018)

[4] C. Li, I.Y. Kim, Multi-material topology optimization for automotive design problems, Proceedings of the Institution of Mechanical Engineers, Part D: Journal of Automobile Engineering 232, 14, 1950-1969 (2018)

[5] K.L. Shenoy, M.S. Kumar, Design topology and electromagnetic field analysis of Permanent Magnet Brushless DC motor for electric scooter application, International Conference on Electrical, Electronics, and Optimization Techniques, ICEEOT 2016, 1541-1545 (2016)

[6] T.I. Petrov, A.R. Safin, I.V. Ivshin, A.N. Tsvetkov, V.Y. Kornilov, The Prospects of Using a Synchronous Machine with Permanent Magnets in the Oil Industry, 14th International 
Scientific-Technical Conference on Actual Problems of Electronic Instrument Engineering, APEIE 2018 - Proceedings, article no. 8546157, 336-338 (2018)

[7] D.N. Dyck, D.A. Lowther, Automated design of magnetic devices by optimizing material distribution, IEEE Trans. Magn. 32, 3, 11881193 (1996)

[8] C.H. Im, H.K. Jun, Y.J. Kim, Hybrid genetic algorithm for electromagnetic topology optimization, IEEE Trans. Magn. 39, 5, 21632169 (2003)

[9] S. Wang, D. Youn, H. Moon, J. Kang, Topology optimization of electromagnetic systems considering magnetization direction, IEEE Trans. Magn. 41, 5, 1808-1811 (2005)

[10] H. Shim, S. Wang, K. Hameyer, Topology optimization of magneto thermal systems considering eddy current as Joule heat, IEEE Trans. Magn. 43, 4, 1617-1620 (2007)

[11] D.H. Kim, J.K. Sykulski, D.A. Lowther, The implications of the use of composite material in electromagnetic device topology and shape optimization, IEEE Trans. Magn. 45, 3, 11541157 (2009)

[12] J.S. Choi, J. Yoo, Structural topology optimization of magnetic actuators using Genetic algorithms and ON/OFF sensitivity, IEEE Trans. Magn. 45, 5, 2276-2279 (2009)

[13] T. Labbe, B. Dehez, Convexity-oriented mapping method for the topology optimization of electromagnetic devices composed of iron and coils," IEEE Trans. Magn. 46, 5, 1177-1185 (2010)

[14] J. Lee, N. Kikuchi, Structural topology optimization of electrical machinery to maximize stiffness with body force distribution, IEEE Trans. Magn. 46, 10, 3790-3794 (2010)

[15] N. Takahashi, T. Yamada, D. Miyagi, Examination of optimal design of IPM motor using ON/OFF method, IEEE Trans. Magn. 46, 8, 3149-3152 (2010)

[16] E.I. Gracheva, O.V. Naumov, A.N. Gorlov, Modelling Characteristics of Reliability LowVoltage Switching Devices on the Basis of Random Checks on the Example of Contactors, Proceedings - 2019 1st International Conference on Control Systems, Mathematical Modelling, Automation and Energy Efficiency, SUMMA 2019, article no. 8947595, 641-643 (2019) 